\title{
Unmasking the linear behaviour of slow motor adaptation to prolonged convergence.
}

\author{
Ian Erkelens ${ }^{1}$, OD (Corresponding author; ierkelen@uwaterloo.ca) \\ Benjamin Thompson ${ }^{1}$, PhD \\ William Bobier ${ }^{1}, \mathrm{OD}, \mathrm{PhD}$ \\ ${ }^{1}$ University of Waterloo School of Optometry \& Vision Science \\ Submitted to: Proceedings of the Royal Society of London B: Biological Sciences \\ October $29^{\text {th }}, 2015$ \\ This is the peer reviewed version of the following article: Erkelens, I. M., Thompson, B., \& Bobier, W. \\ $R$. (2016). Unmasking the linear behaviour of slow motor adaptation to prolonged convergence. \\ European Journal of Neuroscience, 43(12), 1553-1560, which has been published in final form at \\ https://doi.org/10.1111/ejn.13240 This article may be used for non-commercial purposes in \\ accordance with Wiley Terms and Conditions for Self-Archiving.
}




\begin{abstract}
Adaptation to changing environmental demands is central to maintaining optimal motor system function. Current theories suggest that adaptation in both the skeletal-motor and oculomotor systems involves a combination of fast (reflexive) and slow (recalibration) mechanisms. Here we used the oculomotor vergence system as a model to investigate the mechanisms underlying slow motor adaptation. Unlike reaching with the upper limbs, vergence is less susceptible to changes in cognitive strategy that can affect the behaviour of motor adaptation. We tested the hypothesis that mechanisms of slow motor adaptation reflect early neural processing by assessing the linearity of adaptive responses over a large range of stimuli. Using varied disparity stimuli in conflict with accommodation, the slow adaptation of tonic vergence was found to exhibit a linear response whereby the rate and amplitude of the adaptive effects increased proportionally with stimulus amplitude. These results suggest that this slow adaptive mechanism represents an early neural process, implying it is a fundamental physiological process that is potentially dominated by subcortical and cerebellar substrates.
\end{abstract}

\title{
1. Introduction
}

1.1 - Background: Motor adaptation allows for the recalibration of physical responses to changing environmental or sensory demands. This process is essential for maintaining optimal motor control and is present in both the skeletal and oculomotor systems. Deficits in motor adaptation result in inaccurate reaching and saccadic eye movements in disorders such as Parkinson's disease(1). Despite the importance of motor adaptation for accurate and efficient movement of the limbs and eyes, the basic neural mechanisms involved are only beginning to be understood. Converging evidence from human studies suggest that the cerebellum plays a central role in adaption of both the skeletal and oculomotor systems $(2,3)$.

Recently, multiple neural processes have been discovered in the adaptation of reaching movements. These different processes, 'fast' and 'slow', occur simultaneously during adaptation(4). When a reaching movement is perturbated by an external force during its execution, errors occur. 'Fast' adaptive mechanisms provide an immediate but transient response to these errors. 'Slow' mechanisms generate a long-term recalibration of the movement if the perturbation consistently occurs during subsequent movements. The purpose of the slow process is to minimize errors, which in turn optimizes movement(4). These slow processes were discovered through the phenomenon of spontaneous recovery in reaching movements; whereby a conditioned motor response reappears after it was extinguished through extinction training(4). Motor adaption has generally been considered a basic physiological process due to its linear generalizability across differing stimuli and motor tasks $(5,6)$. However, deviations in this linearity to larger perturbations have recently been identified in both reaching and eye movements(7). Complex computational models have been designed to explain these non-linear behaviours; however, this likely reflects the behaviour of both fast and slow adaptive mechanisms combined. Given the complexity of these statistical models, this non-linear behaviour likely represents cognitive, higher level processing. It is reasonable then to assume that non-linearities would exist within such an assessment of motor adaptation due to the cognitive inputs. Separating the contributions of each mechanism to the final adapted response is difficult, which has impeded the study 
of the slower, more elementary neural adaptive processes. To accomplish this it becomes necessary to look for adaptive mechanisms in motor systems with the least complicated neural circuitry.

Eye movements have provided an appropriate model for exploring motor adaptation and can be grouped functionally as either gaze changing or gaze holding. Adaptations of any eye movement can be classified by adjustments in the rapid (fast) neural response driving the eye movement, or by the subsequent change in the levels of tonic innervation that follow the completed movement and hold the eyes in the new position(8). Saccades and vergence are gaze changing eye movements and demonstrate a linear relationship between peak velocity and amplitude of the ballistic portion of the movement $(9,10)$ This is defined as the systems 'main sequence' (MS) (11). It is well established that these responses can be adapted using double-step paradigms(12-14). The recalibration of the fast, open-loop response in saccades occurs quickly (12) (50 trials) if a double-step stimulus is repeated. This adaptation is non-linear to large stimulus amplitudes and the saccadic data (15) seems to also best fit the error relevance model(7). This implies that the adaptation to these double-step paradigms involves a cognitive component, and reflects mainly adaptations in the open-loop, fast systems adaptive responses $(12,14)$. There is little evidence characterizing the adaptive behaviour of the underlying tonic innervations, in isolation, within these motor substrates. It remains unclear if the slower, more basic adaptations in tonic innervations (16) are susceptible to similar cognitive influences seen in the faster systems. The aim of this study was to objectively characterize the properties of a slow adaptive mechanism using the adaptation of tonic vergence within the oculomotor system.

1.2 - Vergence: Ocular vergence is the rotation of the eyes in opposite directions in order to align the visual axes to a new fixation distance, in response to retinal disparity. Convergent disparity is created naturally when an object shifts closer in depth or induced mechanically when horizontal base-out optical prism is placed in front of one eye(17). Vergence is controlled by both fast and slow neural mechanisms with a synkinetic cross-link to ocular accommodation (figure 1A). 'Fast vergence' (FV), comprising both pulse and step innervation, is responsible for generating the complete motor response to a disparity. Vergence also demonstrates a resting or 'tonic vergence' (TV) position, which is seen as the resting angle between the two visual axes after a prolonged period in absolute darkness(8). Adaptations in the TV position can easily be measured by briefly occluding one eye while all other visual information remains fixed. During extended near fixation the FV response fatigues, resulting in errors in the vergence position(18). To avoid this, FV also stimulates the 'slow vergence' system (SV) (19). SV innervation is responsible for adapting the TV position to better match the new fixation distance, which reduces the required FV output (fig. 1B) (20). SV can be considered a slow adaptive process based on its longer decay time constants, which can be in the order of minutes to hours(21). A similar interaction occurs when binocular fixation through a base out optical prism is prolonged. In this instance, the prism does not alter the accommodative demand, which creates a non-congruent, conflicting visual stimulus due to the near triad linkage of vergence and accommodation. This non-congruency generates a much stronger SV response in order to maintain a balance between the accommodation and vergence systems (fig. 1A) $(22,23)$. The ability to easily assess SV innervation in the absence of FV, combined with the large SV response generated under prismatic viewing conditions provide an ideal environment for investigating the response properties of this slower adaptive mechanism. 
1.3 - Purpose: Saccades and FV have been shown to demonstrate positive, linear main sequence effects $(8,10)$. To date, no study has objectively quantified the main sequence characteristics of SV innervation or a slow adaptive process in general, despite the fact that SV is modelled as replacing $F V(19,20)$. We hypothesize that SV will demonstrate linear adaptive effects over a large range of disparity stimuli based upon model predictions of FV, which demonstrates linear MS effects, as the stimulus input to SV(19). If SV were characterized by a positive MS effect, it would suggest that the nature of the SV response is drawn from the FV system. Most importantly, strong linear MS effects over a large stimulus range would suggest this type of adaptation is predominantly an early neural process, whereby cognitive processing is limited. This would suggest this type of adaptation is a fundamental physiological process, likely controlled by the midbrain and cerebellum(24).
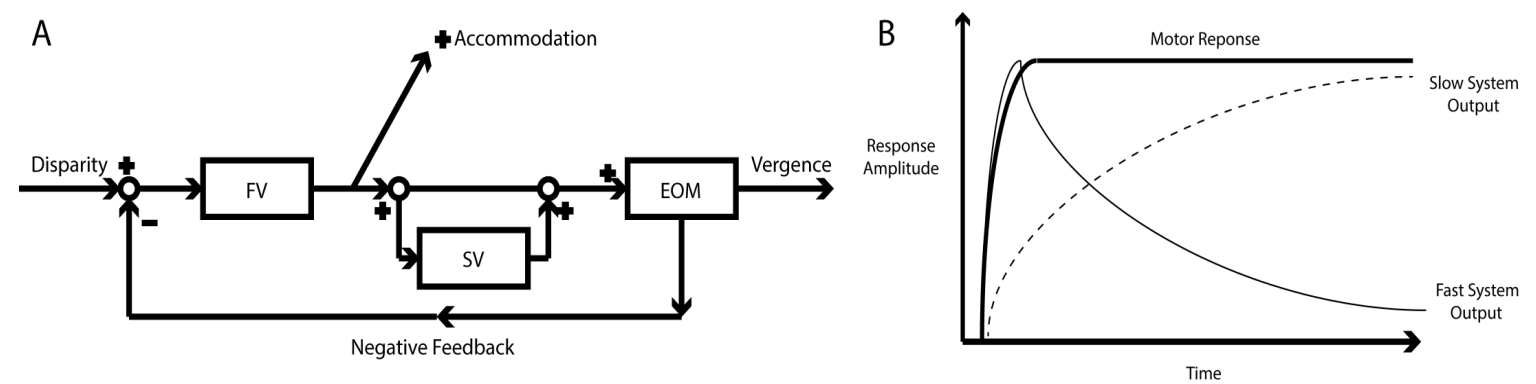

Figure 1. A) Simplified model of vergence control; Fast Vergence (FV) responds to disparity (error in vergence angle) to generate a convergence response. The convergence response also stimulates the accommodative system to increase the ocular focus. TV adaptation is achieved by replacement of the FV controller output by SV. The reduction of FV then uncouples the vergence driven accommodative response. Errors in the vergence position are fed back into the system. (adapted from Schor(19)) B) Graphical representation of a convergence response (bold line) and the contributions of Fast and Slow mechanisms. The Fast response initially drives the movement and then decays as it is gradually replaced by the slow system. The overall response shows little change. (Adapted from Schor(20))

\section{Methods}

2.1 - Overview \& Statement of Ethics: Participants were recruited from the graduate student population at the School of Optometry and Vision Science, University of Waterloo. Informed consent was obtained after verbal and written explanations of the study procedures. The study protocol was approved by the University of Waterloo ethics review board and adhered to the tenets of the Declaration of Helsinki.

\section{2 - Participants: 4 individuals participated. Each was screened by an optometrist to ensure normal} binocular vision $(23,25)$. Visual acuity was $6 / 6$ in each eye and stereopsis was at least 40 seconds of arc. There was no history of ocular surgeries, amblyopia or vision training in any of the participants.

\section{3 - Instrumentation: Vergence stimuli were presented dichoptically via a haploscope. One 7" LCD} monitor was placed at each end of the haploscope arm, $28 \mathrm{~cm}$ from infrared-passing cold mirrors, which were placed orthogonally, $12 \mathrm{~cm}$ from the observer's cornea (figure $2 \mathrm{~A}$ ). The stimuli to each eye were controlled using Experiment Builder ${ }^{\circledR}$ via a host computer. Each eye received a separate white-on-black stimulus with total dimensions of $2.73^{\circ} \times 2.73^{\circ}, 0.08^{\circ}$ stroke width (figure $2 \mathrm{~B}$ ). Each arm of the haploscope was aligned vertically and horizontally to provide a total convergence stimulus of $8.44^{\circ}$ at the cyclopean screen center, with no vertical disparity. This convergence demand is congruent to the 
accommodative demand at the $40 \mathrm{~cm}$ working distance, with an assumed interpupillary distance of $60 \mathrm{~mm}$. This congruent disparity and accommodative stimulus limits any adaptive responses from occurring initially. Additional convergent demands of 4,8 , and $12^{\circ}$ were stimulated by changing the relative position of each target on the monitor, while the accommodative stimulus was held constant. This simulates the same non-congruent disparity provided by base-out prism (figure 2A). Head movements were controlled using a custom chin rest. The apparatus was enclosed in order to control any extraneous peripheral or proximal cues which can affect vergence responses(17).

2.4 - Eye Tracking and Data Analysis: Horizontal eye movements were recorded at $250 \mathrm{~Hz}$ and analyzed

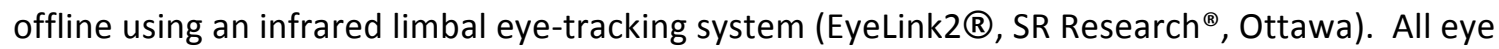
movements were in the linear range of the eye-tracker $\left( \pm 40^{\circ}\right)$. Eye movements were calibrated monocularly at the beginning of each trial using a custom 9-point calibration sequence(26). Left eye and right eye positions were normalized to the screen center as the zero position and converted to degrees from pixel position. The vergence position was taken as the difference between the right and left eye positions relative to the screen center. Blinks and saccades were identified via Data Viewer ${ }^{\circledR}$ and removed using the filter function in Microsoft Excel®.

2.5 - Experimental Protocol (figure 2B): Each participant wore the same corrective lenses, if required, for all sessions. 9 trials were completed, 3 at each non-congruent disparity stimulus amplitude $\left(4^{\circ} 8^{\circ}\right.$ and $12^{\circ}$ ), which was selected randomly at the beginning of each trial. A trial began with 5 minutes of dark adaptation to decay any SV innervation induced by previous visual tasks. The trial was initiated by the researcher, beginning with a brief (2s) presentation of the stimulus to each eye at screen center. The stimulus presented to the right eye was then extinguished to simulate occlusion for 15 seconds, providing a baseline TV measure(27). The right eyes stimulus then reappeared and both left and right stimuli were stepped to an additional $2^{\circ}, 4^{\circ}$ or $6^{\circ}$ convergent position, providing a total retinal disparity of either $4^{\circ}, 8^{\circ}$ or $12^{\circ}$. The subject then viewed this disparity for 15 seconds, before the target of the right eye was extinguished for 15 seconds providing an 'adapted' TV measure. This pattern of binocular fusion interleaved with an adapted TV measure was repeated for 6 minutes using the same noncongruent disparity amplitude for a single trial. Based on previous work, these binocular intervals were selected in order to sufficiently stimulate the SV system $(21,13)$. This design provided a total of 3 minutes of converged binocular viewing with 12 adapted TV measures (figure 2B). Only one trial was completed within in a 8 hour period to prevent fatigue or contamination of any TV adaptation between trials(28).

2.6- Assessing Slow Vergence (SV): Measurement of SV innervation was obtained from the change in TV position measures. A convergent change in the TV position indicated an increase SV innervation output. The SV output was characterized by the change in TV over time in each trial (figure 3A \& B). Previous research has indicated that the decay of FV occurs completely within the first 10 seconds after occlusion. $(22,27)$ Therefore, the TV position was taken as the average of the last 3 seconds of this openloop period.

2.7 - Data \& Statistical Analysis: In each trial for a given convergence stimulus, the TV positions were plotted over time (figure $3 A \& B$ ) and fit with an exponential function using GraphPad $5 \circledR$. An expected asymptotic pattern of TV change resulted, allowing a final amplitude (plateau of the function) and time 
constant $(\tau)$ to be defined for the change in TV due to SV innervation (figure 3B). Maximum velocity (Vmax) of this SV innervation output was calculated for each trial by dividing the plateau amplitude by the time constant $(25,29)$. Each V-max was then plotted against the amplitude of TV change. The linear regression of this data defined the main sequence of slow vergence. The amplitude of SV innervation was plotted over the corresponding disparity stimulus amplitude and a linear regression function was fit for each subject. The amplitude and MS functions of SV innervation are compared between participants using a one-way ANOVA. Linear regressions were fit to each participant. The $r^{2}$ values of these functions were taken as an indicator of the degree of linearity within the system.

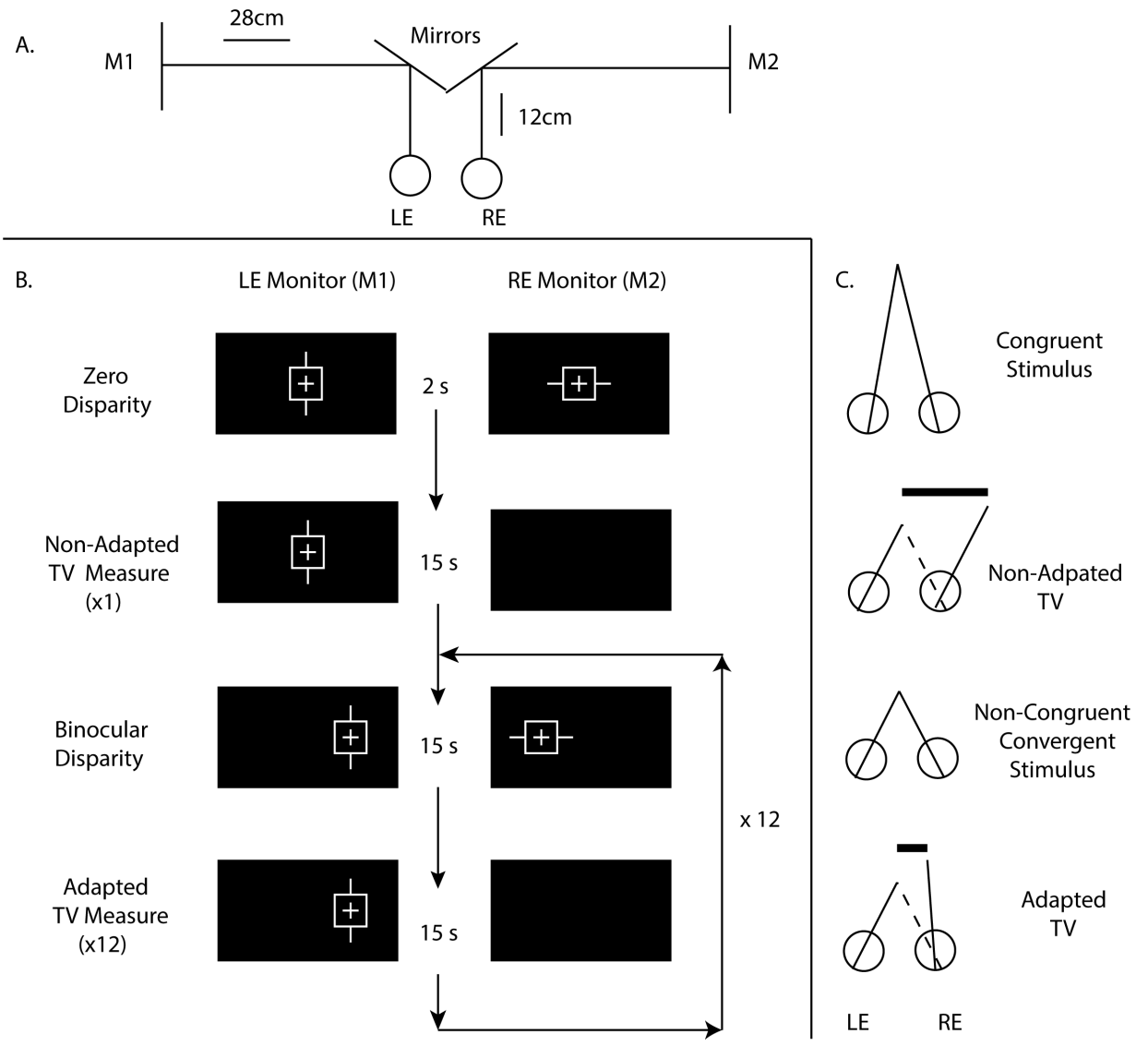

Figure 2. A) Schematic view of the Haploscope (Top, $L E=$ Left Eye, RE = Right Eye). B) Block diagram outlining the stimulus presentation procedure for each trial. C) Schematic of eye position at each stimulus presentation. Solid lines indicate actual eye position. Dashed lines indicate where the fused position of the LE would be during occlusion periods. The angular difference between the dashed and solid line represents the TV position during occlusion. Thick horizontal lines indicate the magnitude (and change) TV due to increased SV output.

\section{Results:}

3.1 Vergence Responses: Subjects were able to fuse all disparity amplitudes using FV. This was shown by a fast vergence movement within $300 \mathrm{~ms}$ of the onset of the disparity stimulus (RE image turned on) and the subject reporting no diplopia. Each time the RE target was extinguished, that eye underwent a fast, divergent movement, indicating a decay of FV output, while the left eye remained stationary. The amplitude of this fast divergent movement reduced overtime, indicating an adaptation of the TV 
position via SV. Figure 3A demonstrates a typical vergence response trace for one complete trial for S1 to a $12^{\circ}$ disparity stimulus. Figure $3 \mathrm{~B}$ shows the same trial (red) when plotted using only TV measures every 15 seconds. The solid line represents the exponential function fit to this data and demonstrates the adaptation of TV via increased output of SV innervation.
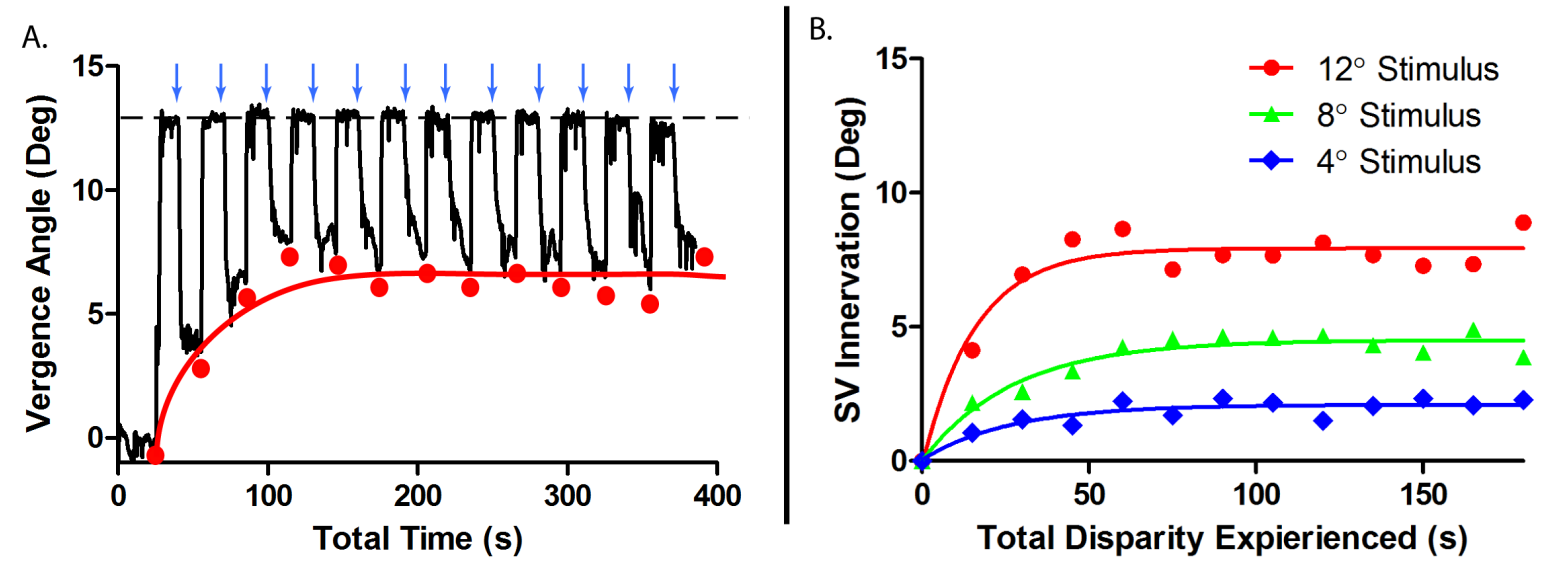

Figure 3. A) Convergence response trace of 1 trial to a $12^{\circ}$ disparity stimulus. The disparity demand of $12^{\circ}$ (dashed line) indicates the fused-adapting position. The TV measurements (circles) rapidly become more convergent after each period of occlusion (arrows), indicating increased SV output. B) The graphical representation of the SV output for the same $12^{\circ}$ trial in $3 A$ (red) and 2 other trials of different stimulus amplitudes (green and blue). Each point represents the average (last 3s) TV position after 15s of occlusion. The exponential function fit to the data is shown, indicating the increased output of SV that is responsible for the convergent adaptation of the TV position.

\section{2 - Amplitude of TV Adaptation: All 4 subjects showed an increase in the amplitude of SV innervation} with increasing disparity amplitudes, indicating that SV adapted the TV position in a linearly proportional manner to the amplitude of the disparity demand (fig. 4A). This positive linear relationship was significant for all 4 subjects (table 1). Comparison of the individual regression functions did not demonstrate a significant difference between the slopes, $F(3,28)=0.67, p=0.58$, or the $y$-intercept, $F(3,31)=0.59, p=0.63$; therefore a combined regression function was defined (table 1 ).

A.

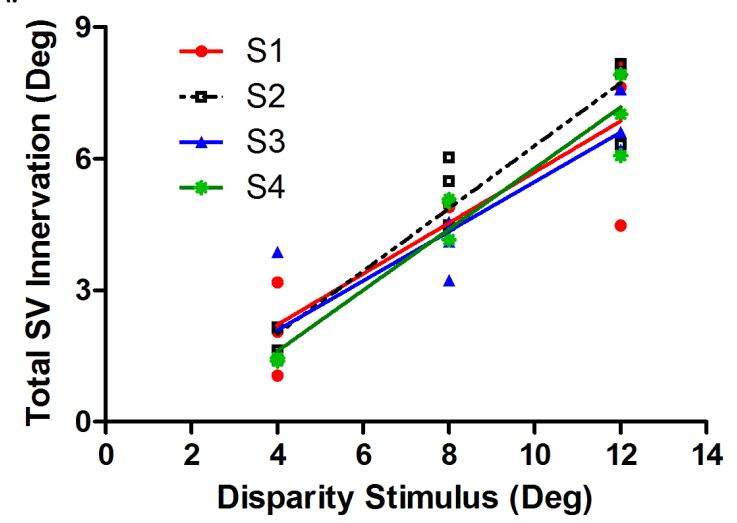

B.

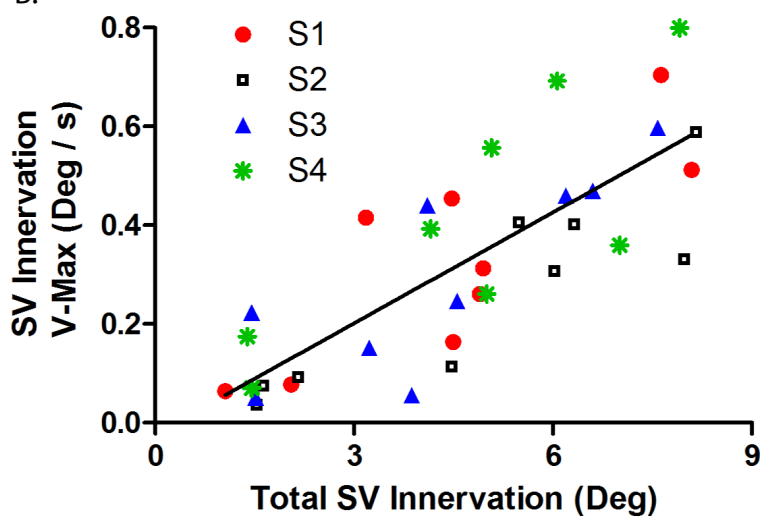

Figure 4. A) Plot of amplitude of SV Innervation output vs. disparity stimulus amplitude for each trial. Y-axis indicates the total change in TV position, indicating the total output of the SV system. Linear regressions are plotted for each subject's data. B) 
Combined Main Sequence plot of V-max of SV vs. SV output amplitude. Each subject demonstrated a significant $(p<0.05)$ positive linear relationship and a strong correlation $\left(r^{2}>0.65\right)$ between rate and amplitude of TV adaptation.

\begin{tabular}{|c|c|c|c|c|c|c|}
\hline Table 1 & \multicolumn{2}{|c|}{ SV Innervation Amplitude (Figure 4A) } & \multicolumn{3}{c|}{ SV Main Sequence (Figure 4B) } \\
\hline Subject & Slope \& Intercept & $\mathrm{R}^{2}$ & $\mathrm{p}$-value & Slope \& Intercept & $\mathrm{R}^{2}$ & $\mathrm{p}$-value \\
\hline S1 & $0.57 \mathrm{x}-0.12$ & 0.76 & 0.0023 & $0.075 \mathrm{x}-0.012$ & 0.67 & 0.0074 \\
\hline S2 & $0.71 \mathrm{x}-0.85$ & 0.92 & $<0.0001$ & $0.066 \mathrm{x}-0.057$ & 0.80 & 0.0013 \\
\hline S3 & $0.56 \mathrm{x}-0.17$ & 0.83 & 0.0007 & $0.076 \mathrm{x}-0.031$ & 0.68 & 0.0065 \\
\hline S4 & $0.70 \mathrm{x}-1.2$ & 0.94 & $<0.0001$ & $0.090 \mathrm{x}-0.020$ & 0.72 & 0.0038 \\
\hline Combined & $0.64 \mathrm{x}-0.046$ & 0.85 & $<0.0001$ & $0.75 \mathrm{x}-0.023$ & 0.65 & $<0.0001$ \\
\hline
\end{tabular}

Table 1. Summary of linear regression analysis of each subjects data and the combined functions for SV Innervation Amplitude and SV innervation Main Sequence (MS).

3.3 - Main Sequence: In all subjects the rate (V-max) of SV innervation output increased linearly with respect to response amplitude (fig $4 \mathrm{~B}$ ). This is demonstrated by the significant positive linear relationship in the MS plots (fig. 4B \& table 1). Comparison of the individual regressions functions did not demonstrate a significant difference in the slope values, $F(3,28)=0.33, p=0.80$ or $y$-intercept, $F(3,31)=2.32, p=0.094$; therefore a combined MS regression function was calculated (table 1 ) and plotted (fig. 4B).

\section{Discussion \&Summary:}

4.1-Discussion: This study provides a novel, objective analysis of the static and dynamic properties of a slow adaptive process in a motor system. Using vergence eye movements and video based eye-tracking, the adaptation of TV via SV was found to exhibit strong linear effects in both its amplitude and rate functions (MS) in all subjects. The significant strength of these linear effects is reflected in the high $r^{2}$ values for the individual and combined functions detailed in table 1. No significant difference was found in these functions between individuals, indicating that a commonly functioning neural mechanism is likely responsible for this adaptive response in binocularly normal individuals. The linear effects seen in SV when compared to previous FV findings $(10,30)$, suggest that the amplitude of the initial disparity error and subsequent motor response of the FV system significantly influence the behaviour of this slow adaptive mechanism. This behaviour of SV is consistent with a number of models that indicate FV as the input signal to the SV system(19,30). In broader motor terms, the (slow) recalibration of this motor system appears to be a function of the initial error amplitude and the subsequent strength of the (fast) corrective movement innervation.

The clear linearity seen in SV innervations adaptive effects on the TV position provides new evidence supporting early processing of this type of slow motor adaptation. Unlike short-term adaptations of subsequent movements to transiently disturbed environments, such as when trying to catch a ball that is randomly blown laterally by a gust of wind, the adaptive responses studied here are generally considered to be reflexively-driven(8). Motor adaptation involving cognitive processes would be expected to distort the expected linearity of the adaptation, especially to large perturbations. Sensory prediction of error has been shown in saccades and limb movements to be central to the learning or adaptation of responses immediately preceding the perturbation induced error $(31,32)$. It has also been shown that the magnitude of the perturbation and cognitive processing of the error relevance 
have varying effects on the linearity of the adaptation of the preceding movements(7). These transient adaptive effects have been used to characterize the fast adaptive processes and are known to demonstrate non-linearity to larger error stimuli $(7,15)$. The strong linearity seen with SV provides empirical evidence supporting the fundamental nature of these slow adaptive mechanisms. The size of the disparity perturbations, with respect to the conflict with accommodation, in this study are significantly larger than what would be experienced naturally at a working distance of $40 \mathrm{~cm}$. If the slow adaptation of TV via SV was to exhibit non-linear effects due to cognitive weighting of the errors relevance to the motor system, we would have expected to see this in both the 8 and $12^{\circ}$ stimuli amplitudes; however, no such deviations from the linear patterns were found in any of the subjects tested. The linearity is then further taken to imply that higher-level cognitive processes are of minimal influence and the relevance of the error is of limited importance to this slow adaptive mechanism.

The linear behaviour of this type of slow adaptation suggests primary influences from subcortical and cerebellar areas. The MS of the slow adapting element in vergence follows a similar MS relationship already established in saccades and FV, but with much lower velocity values $(10,11)$. The cerebellar vermis has been shown to contribute significantly the fast adaptation of saccades to doublestep stimuli(33,34). Various regions of the cerebellum are also known to have significant influence on the dynamic, static and adaptive properties of saccades $(33,35,36)$. Further, cell recordings from the posterior interposed nucleus of the cerebellum show adaptive responses to targets incongruent to demands of accommodation and vergence(37). Therefore, it would not be unreasonable to speculate that the fast and slow adaptive mechanisms of the vergence response result from similar cerebellar activity in conjunction with the midbrain(38). Cell recording studies in of the midbrain and cerebellar vermis in primates have also identified 3 distinct cell types that fire specifically for vergence eye movements $(34,37,39)$. These cells could provide a viable substrate for this adaptation. A simultaneous measure of these neuronal responses patterns with that of FV and SV in future studies could provide empirical evidence to support this hypothesis and would further implicate the cerebellum as a primary site for the modulation of slow adaptive mechanisms.

Clinically, disorders in the motor control of vergence are quite common. Convergence insufficiency $(\mathrm{Cl})$ results in poor convergence responses to target step changes in depth and is estimated to exist in up to $12 \%$ of the general population and roughly $50 \%$ of patients suffering from a traumatic brain injury(40). Research has shown that patients with $\mathrm{Cl}$ possess weak FV and SV responses $(41,42)$. Our data supports the models that indirectly suggest $\mathrm{Cl}$ is a malfunction in the generation of the fast corrective movement in response to disparity $(19,30)$. Which then results in w weakened SV response. Further research exploring the relationship between FV and SV behaviour in patients with $\mathrm{Cl}$ can provide an understanding how these and other adaptive processes fail to develop, malfunction or are impaired such as in $\mathrm{Cl}$ cases resulting from TBI's.

4.2 - Summary: Our data provides a novel assessment of slow motor adaptation measured in ocular vergence. Strong linearity was found in the amplitude and rate of TV adaptation via SV innervation, suggesting this mechanism is a basic physiological function, reflecting early processing that has limited cognitive inputs. The data also suggests that clinical adaptive disorders are an expression of a weak reflexive response that fails to generate an appropriate slow recalibration of the motor system. We 
suggest a theoretical neural origin for these slow adaptive processes based on previous neurophysiological data. Further research utilizing the techniques described within this study can further expand our neural understanding of how different types of motor adaptation occur and how they dysfunction or are impaired through injury.

5.1 - Data Accessibility: The datasets supporting this article have been uploaded as part of the supplementary material.

6.1-Competing Interests: The authors have no competing interests to report.

7.1-Author's Contributions: IE conceived the experiment, collected and analysed the data and drafted the article. All authors contributed to the study design, data interpretations, editing and revising the article. All authors have given final approval of the version to be published.

8.1-Acknowledgements: The authors wish to acknowledge the significant time commitment and support of all 4 participants in the study and the support of the students in the Dr. Bobier Laboratory at the University of Waterloo.

9.1 - Funding: Funding for this project was provided by the University of Waterloo NSERC, COETF and the Ontario QE II GSST fund.

10.1 - Supplementary Data: Supporting data files for this study can be found at:

http://hdl.handle.net/10864/11164 


\section{$\underline{\text { References }}$}

1. Pfeiffer RF, Bodis-Wollner I, editors. Parkinson's Disease and Nonmotor Dysfunction. Totowa, NJ: Humana Press; 2013.

2. Robinson FR, Fuchs AF. The role of the cerebellum in voluntary eye movements. Annu Rev Neurosci. Annual Reviews 4139 El Camino Way, P.O. Box 10139, Palo Alto, CA 94303-0139, USA; 2001 Jan 28;24:981-1004.

3. Houk J, Buckingham J, Barto A. Models of the cerebellum and motor learning. Behav Brain Sci. 1996 Sep;19(3):368-83.

4. Shadmehr R, Smith MA, Krakauer JW. Error correction, sensory prediction, and adaptation in motor control. Annu Rev Neurosci. Annual Reviews; 2010 Jan;33:89-108.

5. Joiner WM, Ajayi O, Sing GC, Smith MA. Linear hypergeneralization of learned dynamics across movement speeds reveals anisotropic, gain-encoding primitives for motor adaptation. J Neurophysiol. 2011;105(1):45-59.

6. Goodbody SJ, Wolpert DM. Temporal and amplitude generalization in motor learning. J Neurophysiol. 1998;79(4):1825-38.

7. Wei K, Körding K. Relevance of error: what drives motor adaptation? J Neurophysiol. 2009 Feb;101(2):655-64.

8. Leigh RJ, Zee DS. The Neurology of Eye Movements. Oxford University Press; 2006. p.286-310.

9. Hung GK, Zhu H, Ciuffreda KJ. Convergence and divergence exhibit different response characteristics to symmetric stimuli. Vision Res. 1997;37(9):1197-205. 
10. Semmlow JL, Hung GK, Horng JL, Ciuffreda KJ. Disparity vergence eye movements exhibit preprogrammed motor control. Vision Res. 1994 May;34(10):1335-43.

11. Bahill AT, Clark MR, Stark L. The main sequence, a tool for studying human eye movements. Math Biosci. 1975 Jan 20;24(3-4):191-204.

12. Semmlow JL, Gauthier GM, Vercher JL. Mechanisms of short-term saccadic adaptation. J Exp Psychol Hum Percept Perform. 1989 May;15(2):249-58.

13. Kim EH, Vicci VR, Granger-Donetti B, Alvarez TL. Short-term adaptations of the dynamic disparity vergence and phoria systems. Exp brain Res. 2011 Jul;212(2):267-78.

14. Munoz P, Semmlow JL, Yuan W, Alvarez TL. Short term modification of disparity vergence eye movements. Vision Res. 1999 May;39(9):1695-705.

15. Robinson FR, Noto CT, Bevans SE. Effect of visual error size on saccade adaptation in monkey. J Neurophysiol. 2003 Aug;90(2):1235-44.

16. Rosenfield M. Tonic vergence and vergence adaptation. Optom Vis Sci. Lippincott Williams \& Wilkins; 1997;74(5):303-28.

17. Schor CM, Ciuffreda KJ. Vergence eye movements: basic and clinical aspects. Butterworth; 1983. p. 65-93, 109-35.

18. Schor C. Fixation of disparity: a steady state error of disparity-induced vergence. Am J Optom Physiol Opt. 1980 Oct;57(9):618-31.

19. Schor CM. A dynamic model of cross-coupling between accommodation and convergence: simulations of step and frequency responses. Optom Vis Sci. 1992 Apr;69(4):258-69. 
20. Schor $\mathrm{CM}$. The relationship between fusional vergence eye movements and fixation disparity. Vision Res. 1979 Jan;19(12):1359-67.

21. Sethi B. Heterophoria: a vergence adaptive position. Opthalmic and Physiological Optics. 1986;(2):151-6.

22. Schor CM. The influence of rapid prism adaptation upon fixation disparity. Vision Res.1979 Jan;19(7):757-65.

23. Sethi $B$, North $R$ V. Vergence adaptive changes with varying magnitudes of prism-induced disparities and fusional amplitudes. Am J Optom Physiol Opt. 1987;64(4):263-8.

24. Gamlin PD, Yoon K, Zhang H. The role of cerebro-ponto-cerebellar pathways in the control of vergence eye movements. Eye (Lond). Royal College of Ophthalmologists; 1996 Jan;10 ( Pt 2)(2):167-71.

25. Kim EH, Vicci VR, Han SJ, Alvarez TL. Sustained fixation induced changes in phoria and convergence peak velocity. PLoS One. 2011 Jan;6(6):e20883.

26. Raveendran RN, Babu RJ, Hess RF, Bobier WR. Transient improvements in fixational stability in strabismic amblyopes following bifoveal fixation and reduced interocular suppression. Ophthalmic Physiol Opt. 2014 Mar;34(2):214-25.

27. Han SJ, Guo Y, Granger-Donetti B, Vicci VR, Alvarez TL. Quantification of heterophoria and phoria adaptation using an automated objective system compared to clinical methods. Ophthalmic Physiol Opt. 2010 Jan;30(1):95-107.

28. Sethi B. Vergence adaptation: a review. Doc Ophthalmol. 1986;63(3):247-63.

29. Kim EH, Alvarez TL. The Changes in Phoria and Convergence to Divergence Peak Velocity Ratio Are Correlated. Curr Eye Res. 2012;37(11):1054-65. 
30. Maxwell J, Tong J, Schor CM. The first and second order dynamics of accommodative convergence and disparity convergence. Vision Res. 2010 Aug 6;50(17):1728-39.

31. Desmurget M, Pélisson D, Urquizar C, Prablanc C, Alexander GE, Grafton ST. Functional anatomy of saccadic adaptation in humans. Nat Neurosci. 1998 Oct;1(6):524-8.

32. Mazzoni P, Krakauer JW. An implicit plan overrides an explicit strategy during visuomotor adaptation. J Neurosci. 2006;26(14):3642-5.

33. Takagi M, Zee DS, Tamargo RJ. Effects of Lesions of the Oculomotor Vermis on Eye Movements in Primate: Saccades. J Neurophysiol. 1998 Oct 1;80(4):1911-31.

34. Nitta T, Akao T, Kurkin S, Fukushima K. Involvement of the cerebellar dorsal vermis in vergence eye movements in monkeys. Cereb cortex. 2008 May;18(5):1042-57.

35. Straube a, Deubel H, Ditterich J, Eggert T. Cerebellar lesions impair rapid saccade amplitude adaptation. Neurology. 2001;57:2105-8.

36. Jenkinson N, Miall RC. Disruption of saccadic adaptation with repetitive transcranial magnetic stimulation of the posterior cerebellum in humans. Cerebellum. 2010;9(4):548-55.

37. Zhang H, Gamlin PD. Neurons in the posterior interposed nucleus of the cerebellum related to vergence and accommodation. I. Steady-state characteristics. J Neurophysiol. 1998;79(3):125569.

38. Collins T, Semroud A, Orriols E, Doré-Mazars K. Saccade dynamics before, during, and after saccadic adaptation in humans. Invest Ophthalmol Vis Sci. 2008;49(2):604-12.

39. Mays LE, Porter JD, Gamlin PD, Tello CA. Neural control of vergence eye movements: neurons encoding vergence velocity. J Neurophysiol. 1986 Oct;56(4):1007-21. 
40. Cooper J, Jamal N. Convergence insufficiency: A major review. Optometry. 2012;83(4):137-58.

41. Sreenivasan V, Bobier WR. Reduced vergence adaptation is associated with a prolonged output of convergence accommodation in convergence insufficiency. Vision Res. 2014 Jul;100:99-104.

42. Daftari A, Alvarez TL, Chua F, Demarco R, Ciufieda K. The Dynamics of Convergence Insufficiency. IEEE 29th Annu Northeast Bioeng Conf. 2003;41-2. 